\title{
Galectin-1 promotes tumor progression via NF- $\kappa B$ signaling pathway in epithelial ovarian cancer
}

\author{
Le Chen ${ }^{1}$, Ying Yao ${ }^{2}$, Lijuan Sun ${ }^{1}$, Jie Tang ${ }^{1 凶}$ \\ 1. Department of Gynecologic Oncology, Hunan Cancer Hospital, the Affiliated Cancer Hospital of Xiangya School of Medicine, Central South University, \\ Changsha, P.R. China. \\ 2. Department of Gynecology and Obstetrics, the First People's Hospital of Yueyang, Yueyang, P.R. China.
}

$\triangle$ Corresponding author: Jie Tang, MD, Ph.D, Professor, Department of Gynecologic Oncology, Hunan Cancer Hospital, the Affiliated Cancer Hospital of Xiangya School of Medicine, Central South University, Changsha, P.R.China 410006. Address: 283 Tongzipo Road, Yuelu District, Changsha, Hunan, P.R. China 410013. Tel: +86073189762071, Fax: +86073188651800, Email: tanjie_hospital@126.com.

(c) Ivyspring International Publisher. This is an open access article distributed under the terms of the Creative Commons Attribution (CC BY-NC) license (https://creativecommons.org/licenses/by-nc/4.0/). See http://ivyspring.com/terms for full terms and conditions.

Received: 2017.05.01; Accepted: 2017.06.27; Published: 2017.10.17

\begin{abstract}
Purpose: We previously reported that Galectin-1 (Gal-1) played a role in epithelial ovarian cancer (EOC) progression. In this study, we aimed to further investigate the association between Gal-1 expression and prognosis in EOC patients and tried to reveal some novel potential mechanisms of Gal-1 in EOC invasion and migration.

Materials and Methods: Gal- 1 and nucleus NF-кBp65 expression in 109 human epithelial ovarian cancer tissue specimens were evaluated by immunohistochemistry. The Cox model and survival curves were used to investigate the effect of Gal-1 on EOC prognosis. Correlation between Gal-1 expression and NF- $\kappa B$ activation in EOC patients was also analyzed. In vitro experiments were further performed to reveal the function and mechanisms of $\mathrm{Gal}-1$ in invasion and migration of EOC cells.

Results: Expression level of Gal-1 in EOC tissue was an independent prognostic factor on overall survival $(p<0.05)$ and progression-free survival $(p<0.05)$. Patients with high Galectin-1 expression had shorter overall survival $(O S, p<0.05)$ ) and progression-free survival (PFS, $p<0.05)$. Immunohistochemistry revealed that expression of Gal-1 was positively associated with activation of NF- $\kappa$ Bp65 in EOC tissues (Kappa coefficient $=0.458, p<0.001$ ). Patients with tumors concomitantly co-over-expressing Gal-1 and NF- $\kappa B p 65$ had the worse OS $(p<0.001)$ and PFS $(p<0.001)$. The abilities of migration and invasion for EOC cells were significantly reduced after Gal-1 knocked-down in human EOC cell line HO8910, which was accompanied with the suppression of NF-kb pathway activation and with the matrix metalloproteinase- 2 and matrix metalloproteinase- 9 down-regulation.

Conclusions: Our results suggest that Gal-1 is associated with poor outcome in EOC and Galectin-1 promotes tumor progression via NF- $\kappa B$ pathway activation in EOC.
\end{abstract}

Key words: epithelial ovarian cancer; Galectin-1; NF-кB signaling pathway; matrix metalloproteinase-2; matrix metalloproteinase-9; migration and invasion

\section{Background}

Ovarian cancer is the leading cause of death from gynecological malignancies and $90 \%$ of ovarian cancer is epithelial ovarian cancer (EOC) [1]. Debulking surgery and chemotherapy are standard treatment for EOC. However, the 5-year survival rate of EOC is still unsatisfied due to extensive local tumor invasion and metastasis, early systemic dissemination [1]. Thus, understanding the mechanisms which regulates EOC invasion and metastasis is crucial.
Galectins are animal lectins defined by an affinity for $\beta$-galactosides and a highly conserved carbohydrate recognition domain of about 130 amino acids [2]. Galectin-1 (Gal-1) is a 14-kDa homodimeric prototype of galectins laminin-binding galectin encoded by LGALS1 gene (GeneBank Accession\#: NM_002305). Increasing clinical evidences have confirmed that Gal-1 is expressed in many tumor types such as prostate, lung and is associated with a 
poor prognosis in these cancers [3-5]. Gal-1 is involved in many biologic processes, including cell differentiation, tissue development, pre-mRNA splicing, immunoregulation, and tumor progression $[5,6]$.

In our previous study, a significant difference was detected in serum Gal-1 between EOC patients with non-metastatic and those with metastatic disease. It increased in recurrent cases and decreased after debulking surgery. We also found that over expression of Gal-1 significantly increased the migration and invasion abilities of human ovarian cancer cell line OVCAR-3 cells [7]. Thus, we concluded that Gal-1 might play an important role in tumor progression in EOC. In this novel study, we continued to investigate the association between Gal-1 expression and prognosis in EOC patients. Besides, we tried to reveal some novel potential mechanisms of Gal-1 in EOC invasion and migration.

\section{Materials and Methods}

\section{Patients and samples}

All study participants signed an informed consent form which was reviewed by the Institutional Review Board of Hunan Cancer Hospital (Changsha, China). EOC patients were identified from a database containing clinical pathology and follow-up data. All EOC patients were treated with standard protocols in accordance with The National Comprehensive Cancer Network (NCCN) clinical practice guideline for EOC [8] by gynecological oncologists in the Hunan Cancer Hospital between 2008 and 2012. Surgical evaluation was used to determine the clinical stages and presence of metastases according to the 2014 FIGO classification [9], whereas histopathologic analysis was performed by gynecologic pathologists to assess cancer types and grades. The total number of EOC tissues used for tissue microarray (TMA) and immuno-histochemistry (IHC) were 109. Among these cases, 70 were serous carcinoma, 24 were mucinous carcinoma, 10 were endometroid carcinoma, and 5 were clear cell carcinoma. Follow-up of all patients was performed for up to 3 years after surgery.

\section{Antibodies}

Primary antibodies used for IHC and Western Blot were: Gal-1 (Santa Cruz, CA, USA), NF-kBp65 (Cell Signaling Technologies, MA, USA), phospho-IKKa/ $\beta$ (Cell Signaling Technologies, MA, USA), Histone H3 (Abcam plc, Cambridge, MA, USA) and $\beta$-actin (Abcam plc, Cambridge, MA, USA).

\section{TMA and IHC}

Procedures used for TMA and IHC were as we previously described [7]. $4 \mu \mathrm{m}$ sections from TMA were prepared on the microtome and placed on clean, positively charged microscope slides. Slides were incubated overnight with primary antibodies (Gal-1: 1:100; NF-кBp65: 1:200). Antigen-antibody reactions were visualized with 3, 3-diaminobenzidine, the chromogenic substrate for peroxidase, and hematoxylin was used to counter stain the tissue.

Protein expression in each case was rated according to a score that added a scale of intensity of staining to the area of staining as described [7]. More than 10 high-power fields were chosen randomly, and $>1,000$ cells were counted for each section. The intensity of staining was graded on the following scale: 0 , no staining; $1+$, mild staining; $2+$, moderate staining; $3+$, intense staining. The area of staining was assessed as follows: 0, no staining of cells in any microscopic fields; $1+,<30 \%$ of tissue stained positive; $2+$, between $30 \%$ and $60 \%$ stained positive; $3+,>60 \%$ stained positive. The score when summed (extension + intensity) was therefore, from 0 to 6 . A combined staining score (extension + intensity) of $\leq 2$ was considered to be a negative staining (low staining); a score between 3 and 4 was a moderate staining; whereas a score between 5 and 6 was a strong staining.

\section{Cell culture}

The human EOC cell lines HO8910 was purchased from the Type Culture Collection of the Chinese Academy of Sciences (Shanghai, China). Cells were cultured in Dulbecco's modified Eagle's medium (DMEM, Gibco, USA) supplemented with 10\% fetal bovine serum (Gibco, USA). Protocol used for cell culture was as we described previously [7].

\section{RNA Interference}

Human Gal-1 shRNA was designed by Shanghai Genechem Co. LTD. This synthetic oligonucleotide used was: 5'-GCUGCCAGAUGGAUACGAAUUTGGA-3'. It has been proven to be efficient in Gal-1 silencing experiments. To knock down Gal-1 in the HO8910 cancer cell line, cells were seeded in a 6-well tissue culture plate with $2 \times 10^{5}$ / well one day prior to infection. The complete culture solution was replaced by the infection-enhanced solution with $5 \mu \mathrm{g} / \mathrm{ml}$ polybrene (AMRESCO, Houston, Texas, USA), and the packed lentivirus was added to cells with multiplicity of infection (MOI=50). After 16 hours, the lentivirus solution was replaced with complete culture solution. Infected cells were sub-cultured every 5-7 days and the stable transfected cells were selected by puromycin $(1 \mu \mathrm{g} / \mathrm{mL})$. The effect of Gal-1 silencing was detected by Western Blot. 


\section{CCK-8 assay}

Cell proliferation was determined by Cell Counting Kit-8 (CCK-8, Dojindo, Japan). EOC Cells were seeded by a density of $2 \times 10^{3}$ cells/well, and incubated with prepackaged CCK-8 solution at $37^{\circ} \mathrm{C}$ for 3 hours, after that, cultured in DMEM medium with $10 \%$ FBS for 24, 48, and 72 hours. We spectrophotometrically measured the absorbance at $450 \mathrm{~nm}$ of the supernatant by using a plate reader (Bio-Rad Laboratory, Hercules, CA, USA). Cell counts were determined with a calibration curve. A minimum of three independent assays were performed.

\section{Transwell migration and invasion assay}

Transwell systems were used as we previously described [7]. For cell invasion assay, Matrigel (BD Biosciences) was added to the upper surface of a polycarbonic membrane (pore size $8 \mu \mathrm{m}$, Corning, USA) to form a thin gel layer to serve as the ECM. The filter was dried overnight in a laminar hood, and then reconstituted with $100 \mu \mathrm{l}$ of PBS at $37^{\circ} \mathrm{C}$ for $2 \mathrm{~h}$. The upper compartment of the filter contained the treated cells at a density of $5 \times 10^{5}$ cells/well in $100 \mu l$ of DMEM. The bottom filter was filled with $600 \mu \mathrm{l}$ of conditional medium. For TNF- $\alpha$-induced NF- $\kappa B$ signaling activation, the bottom filter was additionally added with TNF- $\alpha$ (10ng/mL, R\&D Systems, MN, USA) into the conditional medium [10]. After 24-hour incubation at $37{ }^{\circ} \mathrm{C}$ with $5 \% \mathrm{CO}_{2}$, the polycarbonic membrane was fixed with $4 \%$ paraformldehyde for 10 minutes and stained with $0.2 \%$ crystal violet solution. Cells on the upper surface of the filter were removed by wiping with a cotton swab. Cells that had penetrated to the lower surface of the filter were counted under an Olympus microscope in three randomized fields at a magnification of $100 \times$. Cell migration assay was carried out in a transwell filter on membrane filters, which were not coated with Matrigel. A minimum of three independent assays were performed.

\section{Quantitative real-time PCR (qRT-PCR)}

Total RNA from cells was extracted using the Trizol Kit (Life Technologies, Rockville, MD, USA). QRT-PCR was performed using Accurate Genomic DNA Removal kit (Applied Biological Materials Inc, BC, Canada) and SYBR® Green Realtime PCR Master Mix kit (Takara Bio, Tokyo, Japan). The data in qRT-PCR was analyzed using the $\Delta \Delta \mathrm{Ct}$ method and normalized to $\beta$-actin levels. Primer sequences used in qRT-PCR were: Gal-1 forward primer: 5'-CTCCTGACGCTAAGAGCTTCG-3', reverse primer: 5'-CCAGGCTGGAAGGGAAAGAC-3'; MMP-2 forward primer: 5'-TGGGAGCATGGCGATGGATA-3', reverse primer: 5'-ACAGTGGACATGGCGGTCTC-3'; MMP-9 forward primer: 5'-TTGACAGCGACAAGAAGTGG-3', MMP-9 reverse primer: 5'-GCCATTCACGTCGTCCTTAT-3'. $\beta$-actin forward primer 5'-CCTGGCACCCAGCACAAT-3', reverse primer 5'-GGGCCGGACTCGTCATCG-3'. A minimum of three independent assays were performed.

\section{Western Blot}

A summary of our Western Blot protocol [7] is as follows. The cell lysate used for Western Blot was extracted within RIPA lysis buffer (Cwbio, Beijing, China), which contains protease inhibitors (Roche, Germany). We quantified proteins by using the BCA Protein Assay Kit (Cwbio, Beijing, China). The Western blot system was established by using a BioRad Bis-Tris Gel system (Bio-Rad, CA, USA). Briefly, we separated approximately $40 \mu \mathrm{g}$ of protein on SDS-PAGE and transferred it to PVDF membranes (Millipore, Danvers, MA, USA). The membranes were blocked by $5 \%$ nonfat milk. Then, the membranes were incubated overnight with the primary antibody (1:100 for Gal-1, 1:1000 for NF-кBp65 and 1:500 for phospho-IKKa $/ \beta$ ) at $4^{\circ} \mathrm{C}$, followed by a brief wash with PBST and subsequent incubation with a secondary antibody for 1 hour at $37^{\circ} \mathrm{C}$. Finally, ECL solution (Millipore, Billerica, MA, USA) was added to cover the blot surface. The signals were captured and the intensity of the bands was quantified by using the Bio-Rad ChemiDoc XRS+ system (Bio-Rad, CA, USA). At least three independent experiments were performed.

\section{Statistical analysis}

All quantitative data were presented as mean \pm SEM values. Data from two groups were compared using Student's $\mathrm{T}$ test. The Kappa coefficient was used to analyze the correlation between Gal-1 expression and NF-кBp65 activation. Cumulative survival rate was calculated with the Kaplan-Meier method, and the significance of differences in survival rates was analyzed by the log-rank test. Cox proportional hazard models were used to assess the prognostic significance of Gal-1 expression and several clinicopathologic parameters. $p<0.05$ was considered statistically significant. Computations were performed using the SPSS 18.0 (IBM, Armonk, NY, USA).

\section{Results}

\section{High Gal-1 expression in EOC tissues correlated with poor prognosis}

We previously found that Gal-1 was significantly up-regulated in EOC patients, and this higher Gal-1 
expression was positively correlated with advanced stage and recurrence [7]. To further confirm the relationship between Gal-1 expression and clinical outcomes, 109 EOC cases were divided into two groups (Negative/Low group and Moderate/High group) based on Gal-1 expression determined by the IHC score, which had been previously described [7]. Using univariate analysis (Cox's proportional hazards model), the following variables were found to be significantly associated with overall-survival (OS) and progression-free-survival (PFS): FIGO stages, tumor grades, residual tumor volume and expression levels of Gal-1 ( $p<0.05$; Table 1 and Table 2). Survival curves calculated by Kaplan-Meier method and analyzed by log-rank test showed that the survival rates in the low Gal-1 expression group was significantly longer than that in high Gal-1 expression group (OS: Figure 1A and PFS: Figure 1B, $p<0.05)$. Multivariate analysis showed that FIGO stages, tumor grades, residual tumor volume and expression levels of Gal-1 had an independent prognostic effect on OS ( $p<0.05$; Table 1$)$ and PFS ( $p<0.05$; Table 2).

\section{Expression of Gal-1 was positively associated with the activation of NF-KBp65 in EOC tissues}

Compelling evidence suggests that NF- $\mathrm{kB}$ pathway plays a critical role in ovarian cancer [11]. Since translocation of NF-kBp65 to the nucleus is considered as the essential features in NF- $\mathrm{kB}$ signaling pathway activation [12], we next analyzed NF-kBp65 nuclei expression by IHC in these 109 EOC cases. NF$\kappa \mathrm{B}$ nucleus expression was negative in 17 of EOC samples $(15.60 \%)$ and positive in the remaining 92 samples $(84.40 \%)$. In these 92 cases, 14 samples were low expression $(12.84 \%), 63$ samples were moderate expression $(57.80 \%)$, and the left 15 samples were high expression $(13.76 \%)$. We then investigated the association between Gal-1 expression and the activity of NF-kB p65. As shown in Table 3, Figure 2A and
Figure 2B), Gal-1 expression was positively associated with NF- $\kappa B$ p65 activation $(p<0.001)$. These results collectively suggest that Gal-1 might be closely associated with NF-kBp65 activation in EOC tissues.

Table 1. Prognostic factors on overall- survival were analyzed by univariate and multivariate Cox's proportional hazards models.

\begin{tabular}{lllll}
\hline & \multicolumn{2}{l}{ Univariate analysis } & \multicolumn{2}{l}{ Multivariate analysis } \\
\cline { 2 - 5 } & HR (95\% CI) & $p$-value & HR (95\% CI) & $p$-value \\
\hline Age (years) ( $\geq 55$ Vs. $<55)$ & $1.04(1.00-1.08)$ & 0.157 & $1.28(1.06-1.42)$ & 0.136 \\
Histology Types (Serous Vs. & $0.68(0.31-1.97)$ & 0.203 & $1.25(0.59-2.48)$ & 0.705 \\
Non-serous & & & & \\
Grade (Low Vs. High) & $2.58(1.36-4.74)$ & 0.029 & $2.35(1.40-4.04)$ & 0.040 \\
FIGO Stage (I-II Vs.III-IV) & $5.21(1.88-11.92)$ & $<0.001$ & $4.45(1.28-9.45)$ & $<0.001$ \\
$\begin{array}{l}\text { Residual Tumor (<1 cm } \\
\text { Vs. } \geq 1 \mathrm{~cm})\end{array}$ & $2.79(1.58-3.94)$ & 0.002 & $2.54(1.42-3.86)$ & 0.004 \\
Gal-1 (Negative/LowVs. & $2.27(1.54-3.37)$ & 0.034 & $2.15(1.36-2.77)$ & 0.029 \\
Moderate /High) & & & & \\
\hline
\end{tabular}

Table 2. Prognostic factors on progression-free-survival were analyzed by univariate and multivariate Cox's proportional hazards models.

\begin{tabular}{|c|c|c|c|c|}
\hline & \multicolumn{2}{|c|}{ Univariate analysis } & \multicolumn{2}{|c|}{ Multivariate analysis } \\
\hline & HR (95\% CI) & $p$-value & HR $(95 \%$ CI) & $p$-value \\
\hline Age (years) $(\geq 55$ Vs. $<55)$ & $0.97(0.72-1.69)$ & 0.360 & $1.22(0.77-1.68)$ & 0.456 \\
\hline $\begin{array}{l}\text { Histology Types (Serous Vs. } \\
\text { Non-serous) }\end{array}$ & $0.85(0.61-1.77)$ & 0.216 & $1.35(0.92-2.17)$ & 0.704 \\
\hline Grade (Low Vs. High) & $1.58(0.96-3.74)$ & 0.089 & $1.79(1.48-3.04)$ & 0.061 \\
\hline FIGO Stage (I-II Vs. III-IV) & $4.21(2.37-9.71)$ & $<0.001$ & $3.94(2.32-8.95)$ & $<0.001$ \\
\hline $\begin{array}{l}\text { Residual Tumor }(<1 \mathrm{~cm} \\
\text { Vs. } \geq 1 \mathrm{~cm})\end{array}$ & $3.79(2.08-4.94)$ & 0.001 & $3.45(2.78-4.56)$ & 0.001 \\
\hline $\begin{array}{l}\text { Gal-1 (Negative / Low Vs. } \\
\text { Moderate / High ) }\end{array}$ & $2.82(1.55-4.54)$ & 0.049 & $2.48(1.46-4.77)$ & 0.042 \\
\hline
\end{tabular}

Table 3. Correlation between Gal-1 and NF- $\kappa B$ expression in EOC tissues

\begin{tabular}{lllll}
\hline & \multicolumn{2}{c}{ NF-kB } & p-Value \\
\cline { 2 - 4 } Gal-1 & $\begin{array}{l}\text { Moderate } \\
\text { /High }(\mathrm{n}=78)\end{array}$ & $\begin{array}{l}\text { Negative } \\
\text { /Low }(\mathrm{n}=31)\end{array}$ & $\begin{array}{l}\text { Kappa } \\
\text { coefficient }\end{array}$ & 0en \\
\hline $\begin{array}{l}\text { Moderate /High } \\
(\mathrm{n}=91)\end{array}$ & 74 & 17 & 0.458 & 0.000 \\
$\begin{array}{l}\text { Negative /Low } \\
(\mathrm{n}=18)\end{array}$ & 4 & 14 & & \\
\hline
\end{tabular}

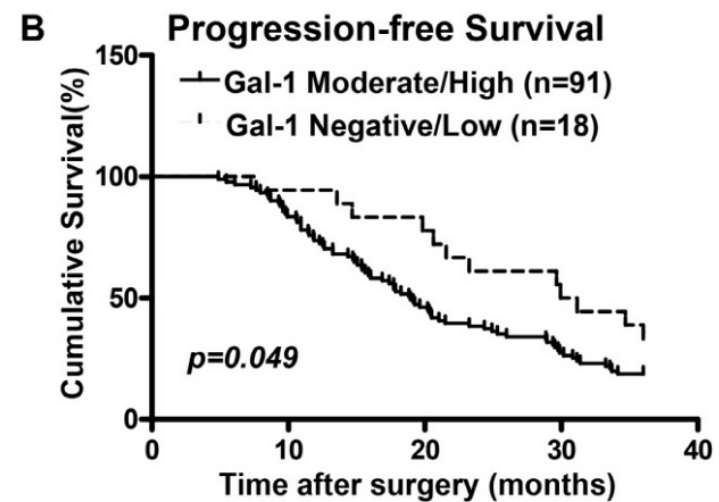

Figure 1. The Kaplan-Meier survival curves compare EOC patients with low and high Gal-1 expression levels. Overall survival rate $(\mathbf{A}$, $p<0.05)$ and progression-free survival rate $(B, p<0.05)$ of the high $\mathrm{Gal}-1$ expressing group $(n=91)$ were significantly lower than these of the low group $(n=18)$. 

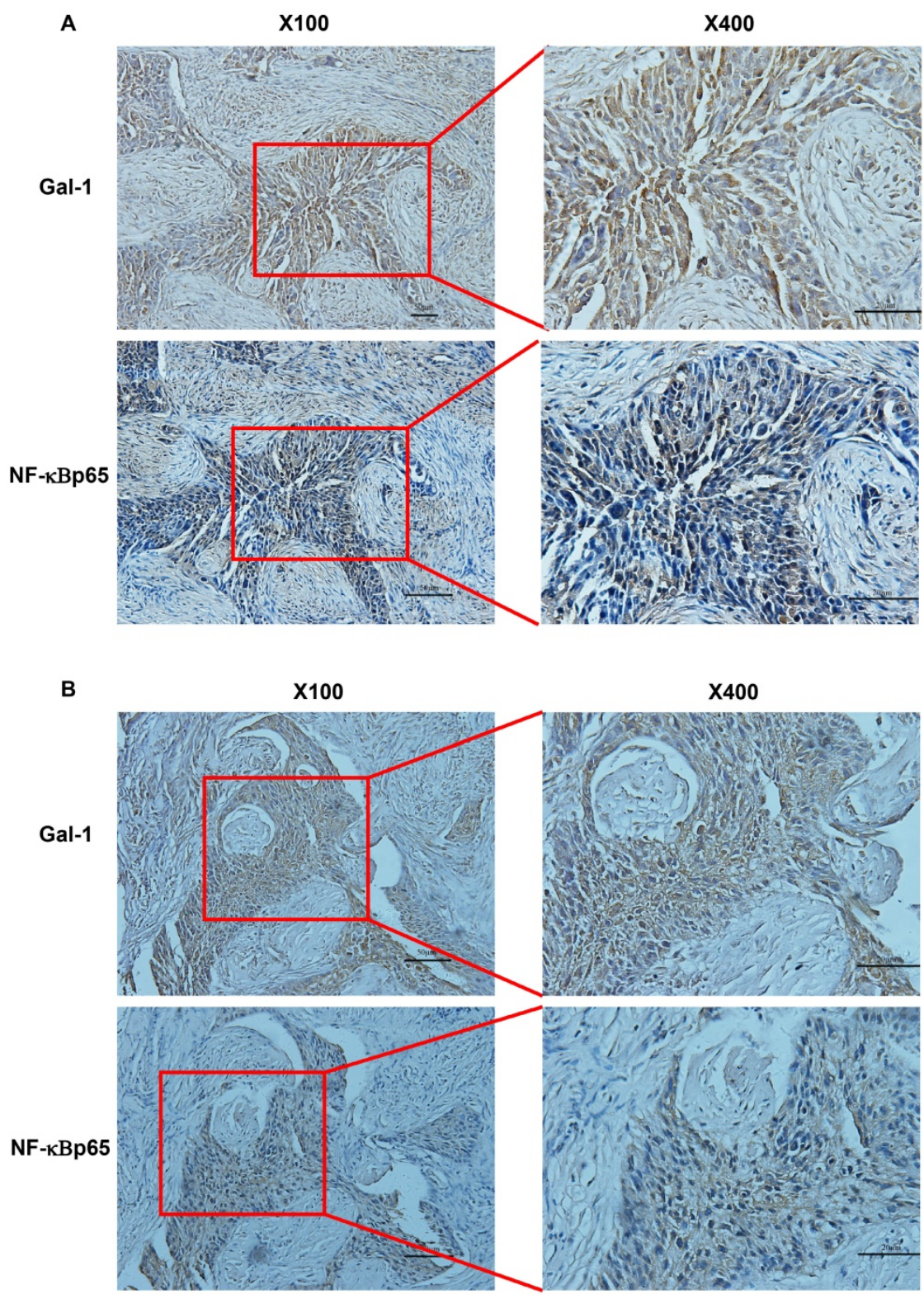

Figure 2. Gal-1 expression and nuclear NF-kBp65 staining in EOC tissues. Serial paraffin TMA sections from same tissues were stained by immunohistochemistry with appropriate antibodies to detect Gal-1 and NF-kBp65 staining. A and B indicated a significant correlation between Gal- 1 expression and the NF-KB activation in the same EOC tissues. Red boxes in the left images indicate the amplified fields presented in the right field. Black scale bars: $50 \mu \mathrm{m}$ for $\times 100,20 \mu \mathrm{m}$ for $\mathrm{X} 400$.

\section{Clinical correlation of Gal-1/ NF-kBp65 co-expression and patient outcome}

Given that expression of Gal-1 was positively associated with the activation of NF- $\mathrm{KBp} 65$ in EOC tissues, we further analyzed the prognostic potential of Gal-1 expression combined with NF-кBp65 activation in EOC patients. Kaplan-Meier analysis and log-rank test were conducted to determine OS and PFS between Gal-1/NF- $\mathrm{B}$ co-Moderate/High group and Gal-1/NF-KB non-co-Moderate/High group. Results showed that patients with tumors concomitantly co-over-expressing Gal-1 and NF-kBp65 had the worse OS (Figure 3A, $p<0.001$ ) and PFS (Figure 3B, $p<0.001$ ). These data provide information that Gal-1 and NF-кBp65 co-over-expression might be a predictor of poor outcome in patients with EOC.

\section{Down-regulation of Gal-1 suppressed the activation of NF-KB signaling pathway and decreased MMPs expression in vitro}

The mechanisms of Gal-1 in EOC progression have not been completely understood. The positive association between Gal-1 expression and NF-кBp65 activation in EOC tissues suggested that Gal-1 might be involved in NF-KB signaling pathway regulation. To further confirm this, relative mRNA levels of Gal-1 in four human EOC cell line were firstly evaluated by qRT-PCR. Similar with our previous report [7], 
OVCAR-3 showed a low level of Gal-1, while the mRNA levels of Gal-1 in HO8910, A2780 and SKOV-3 were high (Figure 4A). Considering this, HO8910 was selected and Gal-1 expression was then stable knock-down by sh-Gal-1. Effect of RNA interference was confirmed by Western Blot (Figure 4B).

Nuclear accumulation of NF-kBp65 was assessed after Gal-1 knock-down in HO8910. Phosphorylated $\mathrm{IKKa} / \mathrm{b}(\mathrm{p}-\mathrm{IKKa} / \mathrm{b})$, which was considered as a key molecular in NF-KB signaling pathway $[13,14]$, was also examined. Western Blots revealed that the levels of nuclear p65 and p-IKKa/b were dramatically decreased in Gal-1-knockdown HO8910 cells (Figure $4 \mathrm{C})$. Since the phosphorylation of $\mathrm{IKKa} / \mathrm{b}$ and the translocation of NF-kBp65 to the nucleus are considered as the essential features in NF-KB signaling pathway activation [12], these results suggested that down-regulation of Gal-1 suppressed the activation of NF-KB signaling pathway. Matrix metalloproteinases (MMPs) are reported as downstream targets of the NF- $K B$ signaling pathway $[15,16]$ and are thought to play important roles in EOC invasion [17, 18]. Considering this, MMP-2 and MMP-9, two classical members of the MMP family, were also examined. QRT-PCR analysis revealed that both the levels of MMP-2 and MMP-9 in HO8910 cells were decreased after Gal-1 knock-down (Figure 4D).
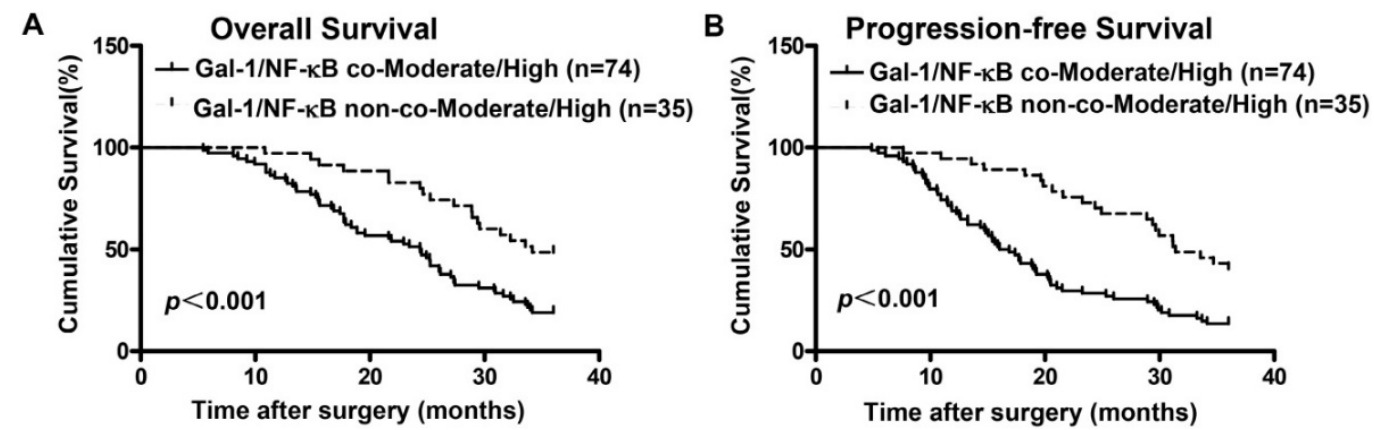

Figure 3. The Kaplan-Meier survival curves compare EOC patients with low and high Gal-1 expression levels. Overall survival rate (A, $p<0.001)$ and progression-free survival rate (B, $p<0.001$ ) of the Gal-1 and NF- kBp65 co-Moderate/High expressing group ( $n=74$ ) were significantly lower than these of the non-co-Moderate/High group $(n=35)$.

A

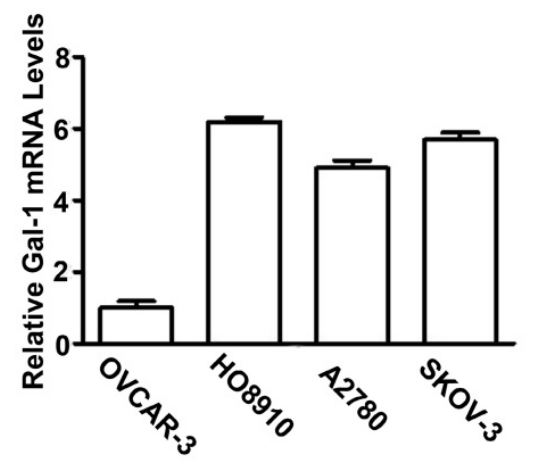

C

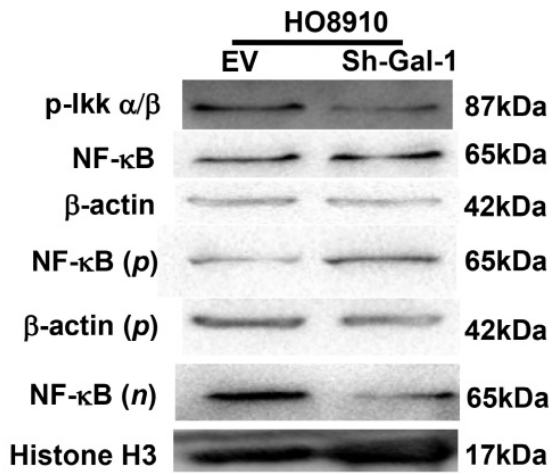

B
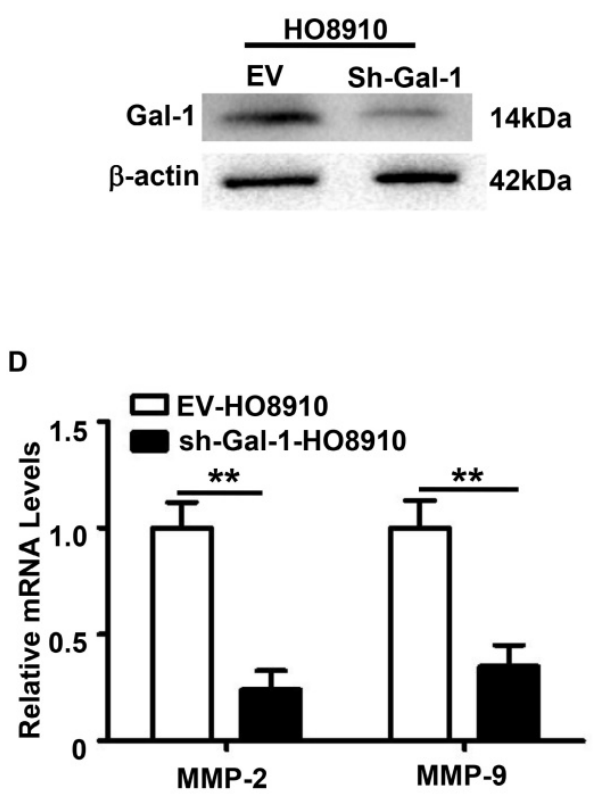

Figure 4. Gal-1 knock-down decreased MMP-2 and MMP-9 through inhibiting the activation of NF-kB signaling pathway in human EOC cell line HO8910. A: QRT-PCR analysis the relative Gal-1 mRNA levels in human EOC cell lines OVCAR-5, HO8910, A2780 and SKOV-3. OVCAR-3 showed a low level of Gal-1, while the mRNA levels of Gal-1 in HO8910, A2780 and SKOV-3 were high. B: Stable down-regulated Gal-1 expression in HO8910 was confirmed by Western Blots. $\beta$-actin served as a loading control. C: Western Blots analysis of the expression levels of the indicated proteins in the HO8910 cells. $\beta$-actin served as a loading control for total and cytoplasm protein, while Histone $\mathrm{H} 3$ was used as a loading control for nuclear protein. $n$ : nucleoproteins; $p$ : plasma protein. D: QRT-PCR indicated that MMP-2 and MMP-9 were both down-regulated after Gal-1 knock-down in HO8910(**p<0.01). 

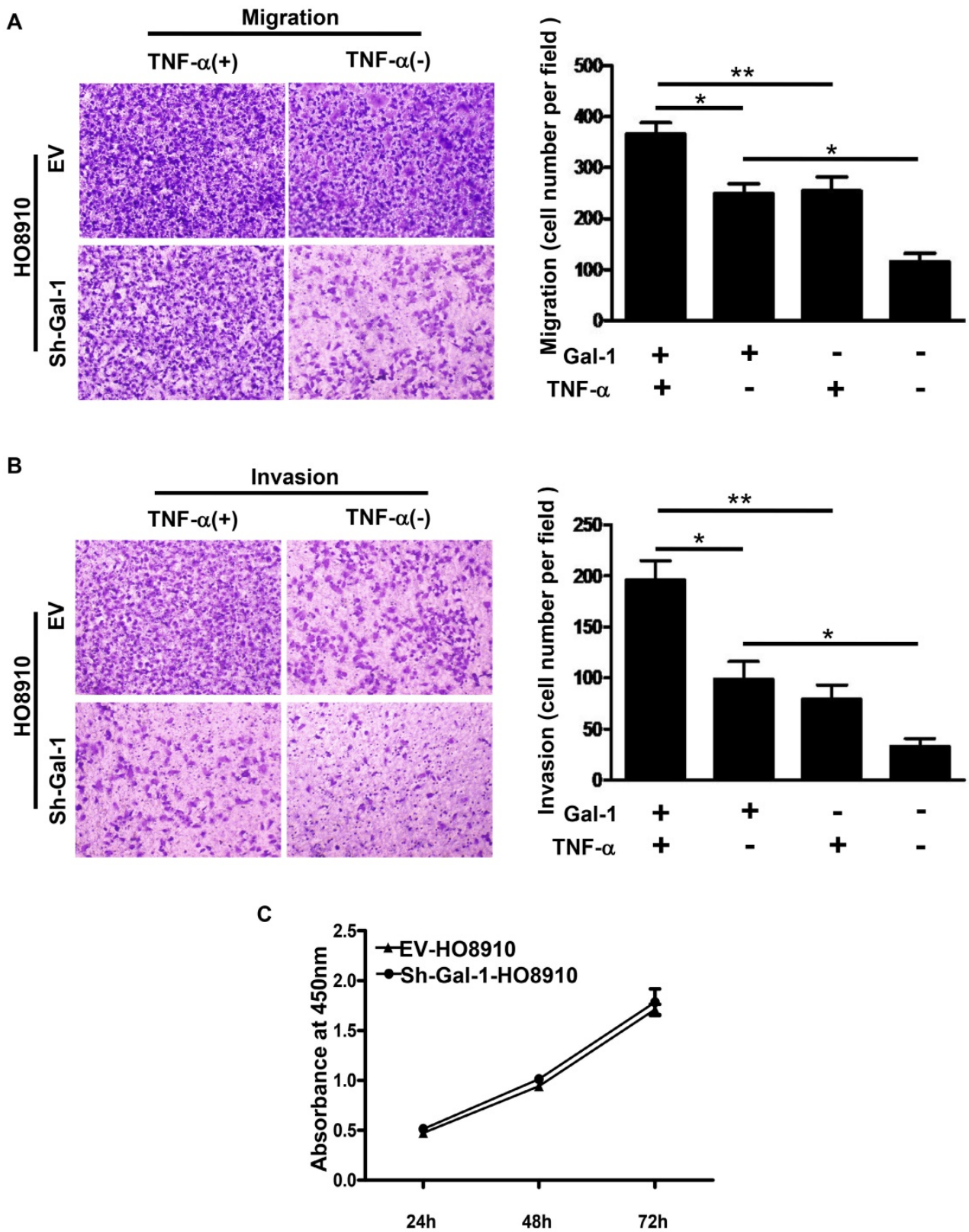

Figure 5. Gal-1 down-regulation suppressed the invasion and migration in human EOC cell line HO8910. A and B: Down-regulation of Gal-1 in HO8910 suppressed migration and invasion abilities of cancer cells significantly. TNF- $\alpha(10 \mathrm{ng} / \mathrm{mL})$ significantly increased cell migration and invasion abilities in $\mathrm{HO} 8910$ cells, but had less effect on the sh-Gal-1-HO8910 cells $\left({ }^{*} p<0.05,{ }^{*} p<0.01\right)$. C: Cell proliferation monitored by CCK-8. Cell proliferation was similar between EV-HO8910 and sh-Gal-1-HO8910 cells $(\mathrm{p}>0.05)$.

\section{Down-regulation of Gal-1 suppressed invasion and migration of EOC cells in vitro}

In our previous study [7], Gal-1 up-regulation significantly increased the migration and invasion abilities of human EOC cell line OVCAR-3. To further confirm the effect of Gal-1 in EOC, Transwell systems were used and we found that the migration and invasion abilities of HO8910 cells were significantly decreased after Gal-1 down-regulation (Figure 5A and 5B). Proliferation abilities between EV-HO8910 cells and sh-Gal-1-HO8910 cells were also evaluated by CCK-8. However, no statistically significant was observed (Figure 5C).

TNF- $\alpha$ is well known to be a proinflammatory cytokine abundantly expressed in ovarian cancer[19, 20] and NF-KB is the primary signaling pathway for TNF $-\alpha$ functions in ovarian cancer cells $[10,21,22]$. To solidify the impact of NF-KB signaling pathway on Gal-1-driven cancer progression, we therefore tested the effects of TNF on cell migration and invasion in HO8910 and sh-Gal-1-HO8910 cells. TNF- $\alpha$, an inducer of NF-kB signaling pathway was used in the transwell system. The results showed that TNF- $\alpha$ significantly increased cell migration and invasion in 
HO8910 cells, but had less effect on the sh-Gal-1-HO8910 cells (Figure 5A and 5B).

\section{Discussion}

Gal-1 expression has been reported to be associated with poor prognosis or positive correlated with tumor invasiveness and higher-grade tumors in several types of cancers $[3,4,6]$. Some articles $[23,24]$ and our previous work [7] demonstrated that Gal-1 was over-expressed in EOC tissues and high levels of Gal-1 in EOC were positively correlated with advanced clinical stage and shorter PFS. In this study, by using Cox's proportional hazards model, we further revealed that Gal-1 expression in EOC tissue was an independent prognostic factor for OS, and high Gal-1 expression was associated with shorter OS in survival analysis.

During metastasis, tumor cells undergo complex changes which modify their cell-cell or cell-ECM adhesion, migration, motility, and invasiveness. There is strong evidence supporting the involvement of Gal-1 in various biological processes linked to tumor cell invasion in hepatocellular [25], pancreatic [26], lung [27], and epithelial ovarian tumors [23, 24], etc. In our previous study, over expression of Gal-1 significantly increased the abilities of OVCAR-3 cells' migration and invasion [7]. In this study, migration and invasion abilities of HO8910 cells were inhibited after Gal-1 knock-down. These results collectively strongly suggest that Gal-1 promotes tumor migration and invasion in human EOC cell lines.

$\mathrm{NF}-\mathrm{kB}$ is an important multifunctional transcription factor, with a wide range of biological activities. Studies have shown high expression of $\mathrm{NF}-\mathrm{kB}$ in various malignant tumors [28-30]. The NF-kB/Rel family consists of five subunits, and the most common dimer of NF-kB is the p50-p65 dimer. In the resting state, the p50-p65 dimer usually directly binds with its inhibitor IKB to form an inactive trimmer, which is present in the cytoplasm of almost all cells. When subjected to stimulation by external factors, such as TNF- $\alpha[10,21,22,31]$, NF-kB firstly dissociates with IKB, which is preceded by the phosphorylation of $\mathrm{IKKa} / \mathrm{b}$, exposing its nuclear localization sequence. The p50-p65 dimer then rapidly translocates from the cytoplasm to the nucleus, and binds with its targeting sequence on the DNA, so as to regulate the transcription of the related gene [12]. Therefore, the expression of p65 can reflect the activity of NF- $\mathrm{KB}$, and the expression of nuclear $\mathrm{NF}-\mathrm{\kappa B}$ protein can be considered a marker of NF- $\mathrm{kB}$ activation [30].

The mechanisms of how Gal-1 promotes tumor progression in EOC have not been completely understood. Recently studies suggested that Gal-1 promotes tumor progression via NF- $\mathrm{KB}$ activation in cancer $[32,33]$. In this study, Gal-1 expression and the activity of NF- $\kappa$ B p65 in compared EOC tissues were evaluated by serial section and IHC, and a significant positively association between these two factors were found. After Gal-1 knock-down in HO8910 cells, a suppression of NF-kBp65 nucleus translocation and a down-regulation of $\mathrm{p}-\mathrm{IKKa} / \mathrm{b}$ were also revealed. Furthermore, we confirmed that TNF- $\alpha$, the best-characterized inducer of NF- $\mathrm{KB}$ signaling pathway, significantly increased cell migration and invasion in HO8910 cells, but had less effect on the sh-Gal-1-HO8910 cells. These results indicated that Gal-1 may promote tumor invasion and migration via the activation of NF- $\mathrm{KB}$ signaling pathway in EOC.

$\mathrm{NF}-\mathrm{kB}$ not only regulates the expression of various chemotactic factors, which promotes cell migration, but also promotes the expression of MMPs, which facilitates invasion and metastasis of tumor cells [33-35]. MMPs are zinc-dependent endopeptidases with well-established roles in the metastasis of many cancer types. Their proteolytic activity directly contributes to the invasion of cancers through extracellular matrix (ECM) and tissue barriers and also activates chemokines and growth factors, which can be further metastasis-promoting. In particular, MMP-2 and MMP-9 are implicated in the spread of EOC, and therefore represents a potential regulator of EOC metastasis. In this current study, level of MMP-2 and MMP-9 were dramatically decreased after Gal-1-knockdown in HO8910 cells. These results collectively strongly suggested that down-regulation of Gal-1 suppressed the activation of NF-kB signaling pathway and decreased MMPs expression.

In summary, in this study, we found that patients with high Gal-1 expression had shorter overall survival (OS) and progression-free survival (PFS). The abilities of migrate and invasion for cancer cells were significantly reduced by down-regulating Gal-1 expression in human EOC cell line HO8910. Furthermore, we revealed that NF-kB pathway and MMPs may be involved in Gal-1 deregulation.

\section{Abbreviations}

EOC: epithelial ovarian cancer; TMA: tissue microarray; IHC: immuno-histochemistry; OS: overall survival; PFS: progression-free survival; MMPs: matrix metalloproteinases; MMP-2: matrix metalloproteinase-2; MMP-9: matrix metalloproteinase-9; TNF- $\alpha$ : tumor necrosis factor- $\alpha$.

\section{Acknowledgements}

We thank all of our patients for participation in this study. 


\section{Funding}

This work was supported by Grant from National Natural Science Foundation of China [grant number 81101996]; the Planned Science and Technology Project of Hunan Province, China [grant number 2014FJ2015]; and the Chinese Anti-Cancer Association [grant number 29].

\section{Competing Interests}

The authors have declared that no competing interest exists.

\section{References}

1. Siegel RL, Miller KD, Jemal A. Cancer statistics, 2016. CA Cancer J Clin. 2016; 66: 7-30.

2. Barondes $\mathrm{SH}$, Castronovo V, Cooper DN, et al. Galectins: a family of animal beta-galactoside-binding lectins. Cell. 1994; 76: 597-8.

3. van den Brule FA, Waltregny D, Castronovo V. Increased expression of galectin-1 in carcinoma-associated stroma predicts poor outcome in prostate carcinoma patients. The Journal of pathology. 2001; 193: 80-7.

4. Szoke T, Kayser K, Baumhakel JD, et al. Prognostic significance of endogenous adhesion/growth-regulatory lectins in lung cancer. Oncology. 2005; 69: 167-74.

5. Astorgues-Xerri L, Riveiro ME, Tijeras-Raballand A, et al. Unraveling galectin-1 as a novel therapeutic target for cancer. Cancer treatment reviews. 2014; 40: 307-19.

6. Cousin JM, Cloninger MJ. The Role of Galectin-1 in Cancer Progression, and Synthetic Multivalent Systems for the Study of Galectin-1. International journal of molecular sciences. 2016; 17.

7. Chen L, Yao Y, Sun L, et al. Clinical implication of the serum galectin-1 expression in epithelial ovarian cancer patients. J Ovarian Res. 2015; 8: 78.

8. National Comprehensive Cancer Network. (NCCN) Clinical Practice Guidelines in Oncology. Ovarian Cancer (Including Fallopian Tube Cancer and Primary Peritoneal Cancer), Version 1.2016. https://www.nccn.org/professionals/physician_gls/f_guidelines.asp\#ovari an. Accessed 15 Feb 2017.

9. Zeppernick F, Meinhold-Heerlein I. The new FIGO staging system for ovarian, fallopian tube, and primary peritoneal cancer. Arch Gynecol Obstet. 2014; 290: 839-42.

10. Dong Y-L, Kabir SM, Lee E-S, Son D-S. CXCR2-Driven Ovarian Cancer Progression Involves Upregulation of Proinflammatory Chemokines by Potentiating NF-kB Activation via EGFR-Transactivated Akt Signaling. PLoS ONE. 2013; 8: e83789.

11. White KL, Rider DN, Kalli KR, Knutson KL, Jarvik GP, Goode EL. Genomics of the NF-kappaB signaling pathway: hypothesized role in ovarian cancer. Cancer causes \& control : CCC. 2011; 22: 785-801.

12. Siebenlist U, Franzoso G, Brown K. Structure, regulation and function of NF-kappa B. Annual review of cell biology. 1994; 10: 405-55.

13. Karin M. How NF-kappaB is activated: the role of the IkappaB kinase (IKK) complex. Oncogene. 1999; 18: 6867-74.

14. Kim HJ, Hawke N, Baldwin AS. NF-kappaB and IKK as therapeutic targets in cancer. Cell death and differentiation. 2006; 13: 738-47.

15. Qin L, Liao L, Redmond A, et al. The AIB1 Oncogene Promotes Breast Cancer Metastasis by Activation of PEA3-Mediated Matrix Metalloproteinase 2 (MMP2) and MMP9 Expression $\square$ Mol Cell Biol. 2008; 28: 5937-50.

16. Park MH, Ahn BH, Hong YK, Min do S. Overexpression of phospholipase D enhances matrix metalloproteinase-2 expression and glioma cell invasion via protein kinase $\mathrm{C}$ and protein kinase A/NF-kappaB/Sp1-mediated signaling pathways. Carcinogenesis. 2009; 30: 356-65.

17. Kenny HA, Kaur S, Coussens LM, Lengyel E. The initial steps of ovarian cancer cell metastasis are mediated by MMP-2 cleavage of vitronectin and fibronectin. J Clin Invest. 2008; 118: 1367-79.

18. Seo JM, Park S, Kim JH. Leukotriene B4 Receptor-2 Promotes Invasiveness and Metastasis of Ovarian Cancer Cells through Signal Transducer and Activator of Transcription 3 (STAT3)-dependent Up-regulation of Matrix Metalloproteinase 2*. J Biol Chem. 2012; 287: 13840-9.

19. Szlosarek PW, Grimshaw MJ, Kulbe H, et al. Expression and regulation of tumor necrosis factor alpha in normal and malignant ovarian epithelium. Molecular cancer therapeutics. 2006; 5: 382-90.

20. Dobrzycka B, Terlikowski SJ, Kowalczuk O, Kinalski M. Circulating levels of TNF-alpha and its soluble receptors in the plasma of patients with epithelial ovarian cancer. European cytokine network. 2009; 20: 131-4.

21. Son DS, Parl AK, Rice VM, Khabele D. Keratinocyte chemoattractant (KC)/human growth-regulated oncogene (GRO) chemokines and pro-inflammatory chemokine networks in mouse and human ovarian epithelial cancer cells. Cancer biology \& therapy. 2007; 6: 1302-12.
22. Son DS, Kabir SM, Dong YL, Lee E, Adunyah SE. Inhibitory effect of tumor suppressor p53 on proinflammatory chemokine expression in ovarian cancer cells by reducing proteasomal degradation of IkappaB. PLoS One. 2012; 7: e51116.

23. Zhang $\mathrm{P}$, Zhang $\mathrm{P}$, Shi B, et al. Galectin-1 overexpression promotes progression and chemoresistance to cisplatin in epithelial ovarian cancer. Cell death \& disease. 2014; 5: e991.

24. Kim HJ, Jeon HK, Cho YJ, et al. High galectin-1 expression correlates with poor prognosis and is involved in epithelial ovarian cancer proliferation and invasion. Eur J Cancer. 2012; 48: 1914-21.

25. Spano D, Russo R, Di Maso V, et al. Galectin-1 and its involvement in hepatocellular carcinoma aggressiveness. Molecular medicine (Cambridge, Mass). 2010; 16: 102-15.

26. Xue X, Lu Z, Tang D, et al. Galectin-1 secreted by activated stellate cells in pancreatic ductal adenocarcinoma stroma promotes proliferation and invasion of pancreatic cancer cells: an in vitro study on the microenvironment of pancreatic ductal adenocarcinoma. Pancreas. 2011; 40: 832-9.

27. Chung LY, Tang SJ, Sun GH, et al. Galectin-1 promotes lung cancer progression and chemoresistance by upregulating p38 MAPK, ERK, and cyclooxygenase-2. Clin Cancer Res. 2012; 18: 4037-47.

28. Ko HS, Lee HJ, Kim SH, Lee EO. Piceatannol suppresses breast cancer cell invasion through the inhibition of MMP-9: involvement of PI3K/AKT and NF-kappaB pathways. Journal of agricultural and food chemistry. 2012; 60: 4083-9.

29. Sasaki N, Morisaki T, Hashizume K, et al. Nuclear factor-kappaB p65 (RelA) transcription factor is constitutively activated in human gastric carcinoma tissue. Clin Cancer Res. 2001; 7: 4136-42.

30. Wang L, Wang C, Jin S, Qu D, Ying H. Expression of NF-kB and PTEN in primary epithelial ovarian carcinoma and the correlation with chemoresistance. Int J Clin Exp Pathol. 2015; 8: 10953-63.

31. Hayden MS, Ghosh S. Regulation of NF-kappaB by TNF family cytokines. Seminars in immunology. 2014; 26: 253-66.

32. Huang CS, Tang SJ, Chung LY, et al. Galectin-1 upregulates CXCR4 to promote tumor progression and poor outcome in kidney cancer. Journal of the American Society of Nephrology : JASN. 2014; 25: 1486-95.

33. Yun SP, Lee SJ, Jung YH, Han HJ. Galectin-1 stimulates motility of human umbilical cord blood-derived mesenchymal stem cells by downregulation of smad2/3-dependent collagen 3/5 and upregulation of NF-kappaB-dependent fibronectin/laminin 5 expression. Cell death \& disease. 2014; 5: e1049.

34. Bilandzic M, Wang Y, Ahmed N, et al. Betaglycan blocks metastatic behaviors in human granulosa cell tumors by suppressing NFkappaB-mediated induction of MMP2. Cancer letters. 2014; 354: 107-14.

35. Trusolino L, Comoglio PM. Scatter-factor and semaphorin receptors: cell signalling for invasive growth. Nat Rev Cancer. 2002; 2: 289-300. 